\title{
Usefulness of non-contrast-enhanced magnetic resonance venography in assessing Klippel-Trenaunay syndrome in an adult patient with renal insufficiency: a case description
}

\author{
Chien-Wei Chen ${ }^{1,2,3,4}$, Kai-Hao Lin ${ }^{1,2}$, Teng-Yao Yang ${ }^{2,5}$, Pang-Yen Chen ${ }^{6,7}$, Yin-Chen Hsu ${ }^{1,2}$, \\ Yao-Kuang Huang ${ }^{2,8}$ \\ ${ }^{1}$ Department of Diagnostic Radiology, Chang Gung Memorial Hospital Chiayi Branch, Chiayi; ${ }^{2}$ Chang Gung University College of Medicine, \\ Taoyuan; ${ }^{3}$ Institute of Medicine, Chung Shan Medical University, Taichung; ${ }^{4}$ Department of Medical Imaging and Radiology, Shu-Zen Junior \\ College of Medicine and Management, Kaohsiung; ${ }^{5}$ Cardiovascular Department, Chang Gung Memorial Hospital Chiayi Branch, Chiayi; \\ ${ }^{6}$ Department of Emergency Medicine, Mackay Memorial Hospital, Taipei; ${ }^{7}$ Institute of Environmental and Occupational Health Sciences, National \\ Yang-Ming University, Taipei; ${ }^{8}$ Division of Thoracic and Cardiovascular Surgery, Wound Center and Plastic Surgery, Chang Gung Memorial \\ Hospital Chiayi Branch, Chiayi
}

Correspondence to: Yao-Kuang Huang, MD, PhD. Chang Gung University College of Medicine, Taoyuan; Division of Thoracic and Cardiovascular Surgery, Wound Center and Plastic Surgery, Chang Gung Memorial Hospital Chiayi Branch, Chiayi. Email: chienwei33@gmail.com.

Submitted Jun 26, 2021. Accepted for publication Oct 29, 2021.

doi: 10.21037/qims-21-671

View this article at: https://dx.doi.org/10.21037/qims-21-671

\section{Introduction}

Klippel-Trenaunay syndrome (KTS) is a congenital vascular anomaly that usually presents in infancy. KTS is primarily diagnosed through clinical evaluation and confirmed by diagnostic imaging using Doppler ultrasound and crosssectional imaging modalities, such as contrast-enhanced computed tomography angiography (CTA) and magnetic resonance angiography (MRA) (1). KTS is rarely diagnosed in adults and can be more difficult to treat than in children due to the added complexity of comorbidities.

One major feature of KTS is a venous malformation, which typically manifests as a distorted (varicose) vein on the surface of the swollen leg. The condition may be accompanied by significant bleeding, deep vein thrombosis (DVT), and embolic complications, leading to chronic stasis ulcers, venous insufficiency, and DVT. For patients with KTS who have persistent serious complications, active treatment, such as surgery or an imaging-guided intervention, may be necessary (2). Precision imaging diagnosis is therefore essential.

Despite ultrasonography being the gold standard for evaluating unilateral leg swelling in clinical practice, it is not the optimal imaging modality for collateral veins and varices. Contrast-enhanced CTA and MRA can be used to image vessels in the entire lower extremity and are important for diagnosing vascular malformations. Noncontrast-enhanced MRA, which has been used to diagnose many diseases (3), may have potential as a diagnostic modality for vascular malformations.

Herein, we report the case of a patient who presented with refractory DVT. With the help of MRA using a triggered non-contrast-enhanced technique, the underlying etiology of the patient's KTS was detected. This paper also discusses the challenges in diagnosing rare vascular pathologies in adults with complex comorbidities and describes the clinical utility and advantages of non-contrastenhanced MRA.

\section{Case presentation}

The patient was a 57-year-old woman with a history of hypertension and chronic kidney disease who had

$\wedge$ ORCID: 0000-0002-5085-9919. 

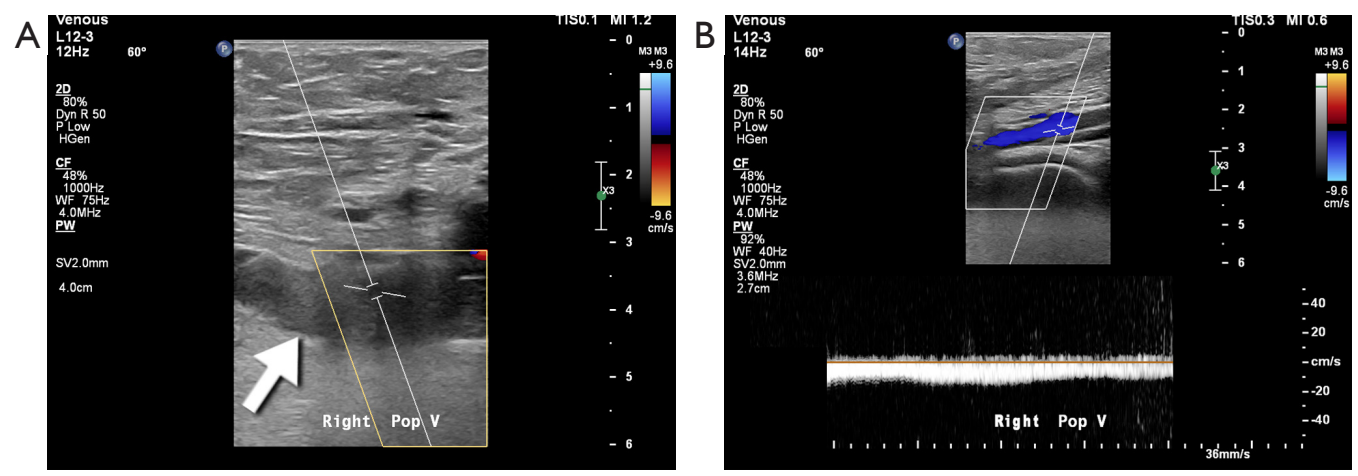

Figure 1 Spectral Doppler ultrasonography in a 57-year-old woman with multiple varicose veins in the right leg. Images show (A) hypoechoic thrombosis (arrow) in the proximal popliteal vein and (B) monophasic waveform in the distal popliteal vein.

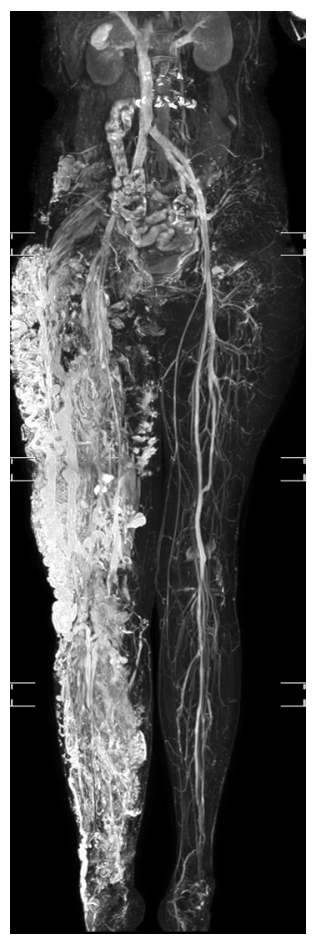

Figure 2 Non-contrast-enhanced MRV. Comparison of bilateral lower limbs revealed extensive venous malformations affecting the right lower limb. A large field of view is one of the advantages of the MRV using TRANCE technique. MRV, magnetic resonance venography; TRANCE, triggered angiography non-contrastenhanced.

suffered from a swollen right leg for many years. Ten years before presenting at our hospital, the patient underwent an ultrasound examination in another clinic and found DVT in her right leg. The patient received long-term anticoagulation (warfarin) treatment and regular follow-up.
Due to increased right leg swelling, she was transferred to our hospital for further evaluation and treatment.

Initial clinical evaluation revealed multiple varicose veins in the patient's right leg. Doppler ultrasound was used as the first-line diagnostic imaging tool, and it revealed right popliteal vein thrombosis and the development of surrounding collateral veins (Figure 1). Considering the possibility of complex venous disease, further imaging studies with a large field of view were needed to assess the entire venous structure. However, during the followup period, the patient's chronic kidney disease rapidly progressed from stage 3 to 4 , rendering contrast-enhanced CTA inappropriate. The use of enhanced MRA also had to be considered carefully. Ultimately, non-contrast-enhanced MRA was scheduled to avoid compromising renal function.

Images were acquired using a turbo spin echo short tau inversion recovery (STIR) sequence during the cardiac systolic phase on a 1.5-Tesla MR scanner (Philips Ingenia, Philips Healthcare, Best, the Netherlands). The STIR sequence provided background suppression (of soft tissue and bone, for example), while the flow-void effect made arterial structures appear dark during the cardiac systolic phase. The three-dimensional (3D) dataset was then collected to reconstruct the bright venous structures in a process known as a triggered angiography non-contrastenhanced (TRANCE) magnetic resonance venography (MRV). Using the MRV protocol (4), it took 25 minutes to acquire images of the entire infradiaphragmatic venous system.

The 3D-MRV revealed multiple tortuous dilated veins in the subcutaneous tissue and leg muscles, even when vessels in the left leg were normal (Figure 2). Hypertrophy associated with a long right leg length was noted. Axial imaging revealed soft-tissue hypertrophy and dilated 

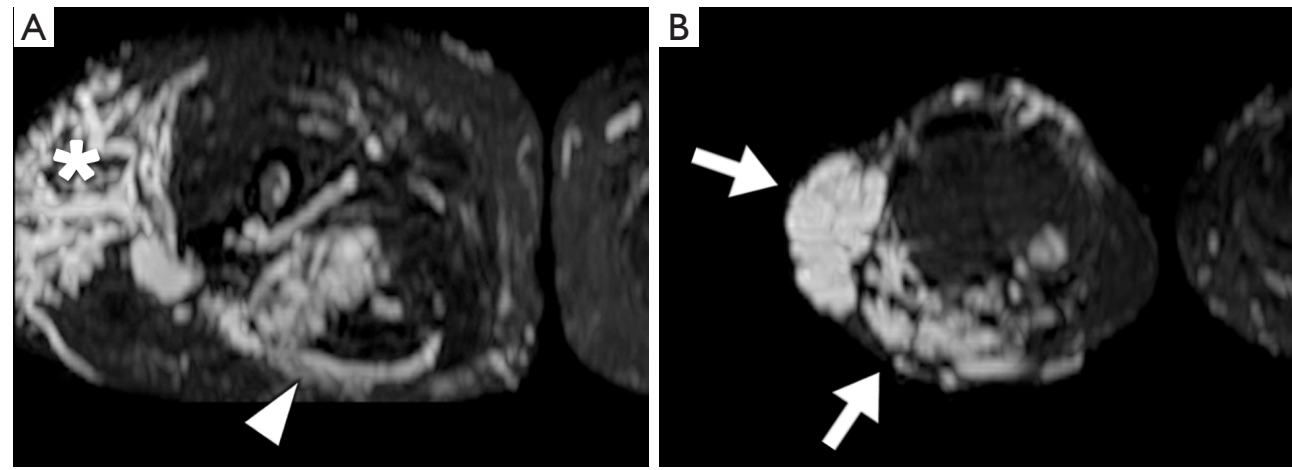

Figure 3 Axial image of the affected lower limb. (A) Presence of the persistent sciatic vein (arrowhead) and the lateral margin veins ${ }^{*}$ ). (B) Dilated vascular channels (arrows) involving the lateral and posterior muscle groups.

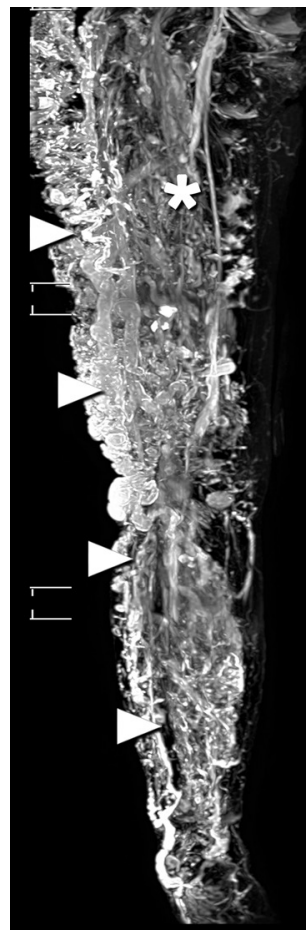

Figure 4 Oblique view of the right lower limb revealing aplasia of the main venous trunk $\left(^{*}\right)$, with venous drainage via marginal veins (arrowheads).

varicose veins. A persistent sciatic vein and a lateral margin vein (Figure 3) were observed, as was femoral vein aplasia with the development of lateral marginal veins (Figures 4,5, Video S1). These imaging findings were consistent with the characteristics of KTS (5). Follow-up after the TRANCEMRV examination confirmed a port-wine stain on the affected leg, which had been missed in the initial clinical evaluation. Precise imaging diagnosis was helpful for preintervention assessment and planning. After discussing with the patient, precise stripping of the collateral veins and reconstruction of the main venous trunk through stent placement were suggested for surgical management. The patient hesitated and opted for follow-up in our hospital with the possibility of surgical treatment in the future.

This study was approved by the Chang Gung Medical Foundation Institutional Review Board (Taoyuan City). All procedures performed in studies involving human participants were in accordance with the ethical standards of the institutional and/or national research committee(s) and with the Helsinki Declaration (as revised in 2013). Written informed consent was obtained from the patient for publication of this case report and accompanying images. A copy of the written consent is available for review by the editorial office of this journal.

\section{Discussion}

KTS is a congenital disease that is usually diagnosed in childhood. However, a small number of cases are diagnosed in adulthood. Owing to the complexity of comorbidities, diagnosing vascular malformations is more difficult in adults than it is in children.

Ultrasonography is usually the first diagnostic tool used for patients with venous conditions involving the lower limbs, especially in patients with renal insufficiency. Patients with chronic kidney disease may be at a higher risk of DVT (6). However, ultrasonography is not particularly sensitive in patients with obesity, edema, or tenderness (7). Further, as ultrasound has a small field of view, using it to assess the entire venous system may be difficult and might 

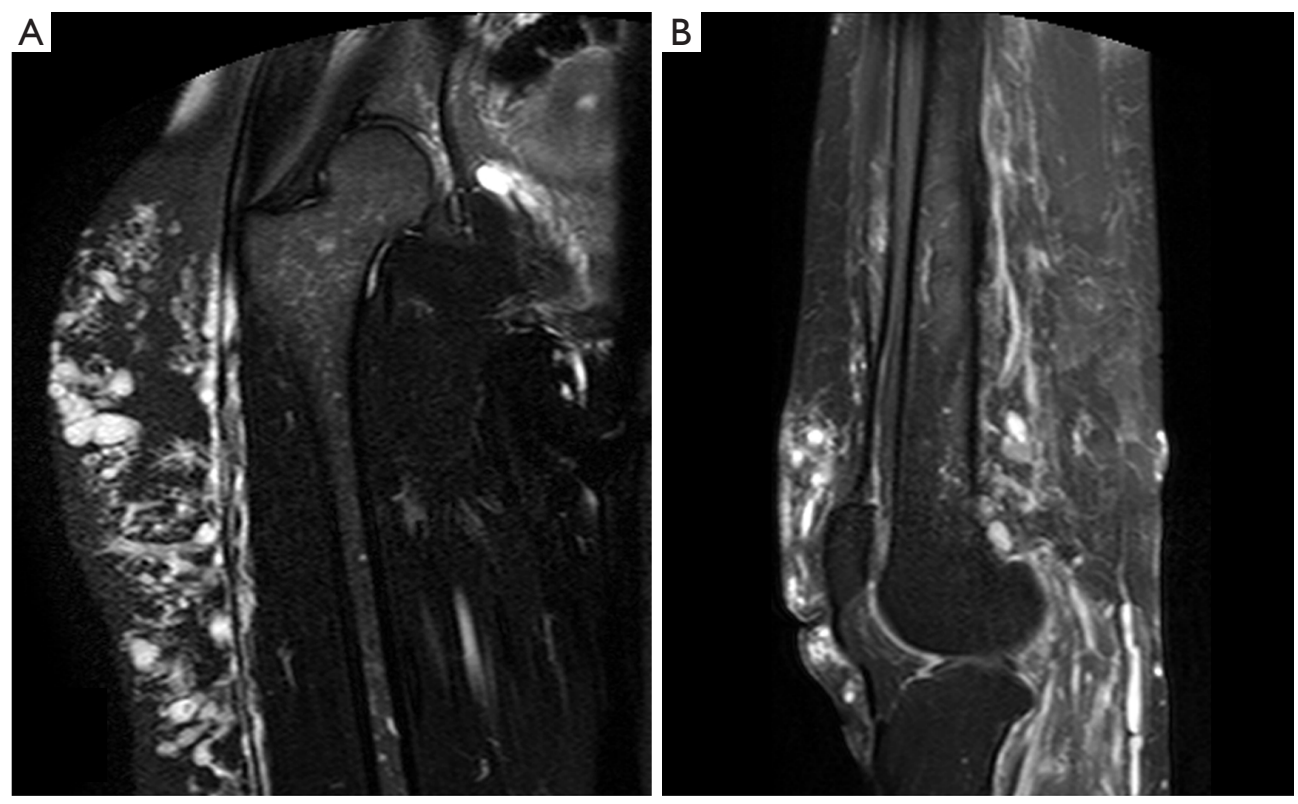

Figure 5 Multiplanar images of the local abnormal blood vessels. (A) Coronal image of the right thigh showing extensive serpentine and dilated vascular canals extending into the subcutaneous soft tissue and muscles. (B) Sagittal image showing downward extension of the vascular malformation to the right knee.

even lead to a misdiagnosis of vascular malformation.

Unlike ultrasound, contrast-enhanced imaging modalities [including computed tomography (CT) or magnetic resonance imaging (MRI) with contrast agents] can be used to assess the entire venous system. They are therefore important for diagnosing vascular malformations. The prevalence reported for these abnormal venous structures varies, but they remain an important feature in the diagnosis of KTS. A previous MRI study reported that the prevalence of persistent sciatic veins in KTS reached $48.8 \%$ (20 out of 41 patients) (8). Another study involving 61 patients with KTS found that the prevalence of lateral venous and deep venous hypoplasia was 53\% (32 patients) and 11\% (7 patients), respectively (5). Intervention for collateral vein dilation in the presence of deep vein hypoplasia can lead to symptoms worsening (9). Moreover, patients with renal insufficiency have a higher risk of developing contrastinduced nephropathy and nephrogenic systemic fibrosis (10). Non-contrast-enhanced MRI can be considered as an alternative modality for diagnosing vascular disease. However, research in this field remains scarce, and most existing studies have focused only on the application of non-contrast-enhanced MRI in arterial diseases (11). The present case report shows that when TRANCE-MRV is used, collateral varicose veins and their territories can be clearly displayed, and venous thrombosis and venous aplasia can be found and distinguished, which is helpful for preintervention assessment and planning.

Ultrasonography is considered to be the first-line imaging tool for the evaluation of lower extremity venous disease. However, diagnostic accuracy and cost-effectiveness are ongoing concerns relating to the use of such diagnostic imaging techniques. A recent study reported substantial inter-rater agreement between ultrasonography and TRANCE-MRV in the diagnosis of DVT. The accuracy of TRANCE-MRV was $88 \%$, and in the 25 ultrasonography negative cases, TRANCE-MRV further detected 16\% (4 cases) DVT, $12 \%$ (3 cases) venous compression, and $4 \%$ (1 case) vena cava anomaly (4). Ultrasonography negative cases may require further vascular surgery if the MRI findings are positive. One of the advantages of TRANCE-MRI is the large field of view, which allows for the evaluation of lesion territory and distribution; such information can prove useful if further vascular surgery is considered. Contrast medium administration increases nephrotoxicity and the risk of nephrogenic systemic fibrosis (10); therefore, we recommend the TRANCE technique as an alternative to contrast-enhanced imaging modalities for the evaluation of patients with renal insufficiency. Furthermore, the TRANCE technique can clearly display the whole 
venous structure with background suppression without contamination of the arterial structure. In contrast, it is difficult to image only the venous structure without the accompanying arterial structure with contrast-enhanced MRI and CT. The main challenge when using these two imaging methods is the difficulty in estimating the correct acquisition time for the optimal contrast opacity of the target vein $(4,12)$.

However, the TRANCE technique also has certain limitations. First, imaging acquisition of the veins in the entire subdiaphragmatic area is time-consuming (taking approximately 25 minutes). Secondly, the technique is not suitable for evaluating patients with cardiac arrhythmias, as inappropriate trigger timing can compromise image quality (13). Finally, the TRANCE technique entails high costs, which must also be considered.

In summary, non-contrast-enhanced MRV can be used to detect vascular malformation in patients with renal insufficiency and is a valuable clinical alternative to contrast-enhanced CT or MRI.

\section{Acknowledgments}

This manuscript was assisted for language editing by the AME Editing Service.

Funding: The study was supported by the Chang Gung Memorial Hospital (CMRPG6K0341, CMRPG6H0651). Chang-Gung Memorial Hospital was responsible for project management, data management, statistics, study registration and was involved in the preparation of the manuscript.

\section{Footnote}

Conflicts of Interest: All authors have completed the ICMJE uniform disclosure form (available at https://dx.doi. org/10.21037/qims-21-671). The authors have no conflicts of interest to declare.

Ethical Statement: The authors are accountable for all aspects of the work in ensuring that questions related to the accuracy or integrity of any part of the work are appropriately investigated and resolved. The study was approved by the Institutional Review Board of Chang Gung Medical Foundation (201900448B0). All procedures performed in studies involving human participants were in accordance with the ethical standards of the institutional and/or national research committee(s) and with the Helsinki Declaration (as revised in 2013). Written informed consent was obtained from the patient for publication of this case report and accompanying images. A copy of the written consent is available for review by the editorial office of this journal.

Open Access Statement: This is an Open Access article distributed in accordance with the Creative Commons Attribution-NonCommercial-NoDerivs 4.0 International License (CC BY-NC-ND 4.0), which permits the noncommercial replication and distribution of the article with the strict proviso that no changes or edits are made and the original work is properly cited (including links to both the formal publication through the relevant DOI and the license). See: https://creativecommons.org/licenses/by-nc-nd/4.0/.

\section{References}

1. Wang SK, Drucker NA, Gupta AK, Marshalleck FE, Dalsing MC. Diagnosis and management of the venous malformations of Klippel-Trénaunay syndrome. J Vasc Surg Venous Lymphat Disord 2017;5:587-95.

2. Gloviczki P, Driscoll DJ. Klippel-Trenaunay syndrome: current management. Phlebology 2007;22:291-8.

3. Miyazaki M, Lee VS. Nonenhanced MR angiography. Radiology 2008;248:20-43.

4. Huang YK, Tseng YH, Lin CH, Tsai YH, Hsu YC, Wang SC, Chen CW. Evaluation of venous pathology of the lower extremities with triggered angiography noncontrast-enhanced magnetic resonance imaging. BMC Med Imaging 2019;19:96.

5. Yamaki T, Konoeda H, Fujisawa D, Ogino K, Osada A, Hamahata A, Nozaki M, Sakurai H. Prevalence of various congenital vascular malformations in patients with KlippelTrenaunay syndrome. J Vasc Surg Venous Lymphat Disord 2013;1:187-93.

6. Lu HY, Liao KM. Increased risk of deep vein thrombosis in end-stage renal disease patients. BMC Nephrol 2018;19:204.

7. Karande GY, Hedgire SS, Sanchez Y, Baliyan V, Mishra V, Ganguli S, Prabhakar AM. Advanced imaging in acute and chronic deep vein thrombosis. Cardiovasc Diagn Ther 2016;6:493-507.

8. Cherry KJ, Gloviczki P, Stanson AW. Persistent sciatic vein: diagnosis and treatment of a rare condition. J Vasc Surg 1996;23:490-7.

9. Gloviczki P, Stanson AW, Stickler GB, Johnson CM, Toomey BJ, Meland NB, Rooke TW, Cherry KJ Jr. Klippel-Trenaunay syndrome: the risks and benefits of 
vascular interventions. Surgery 1991;110:469-79.

10. Kaewlai R, Abujudeh H. Nephrogenic systemic fibrosis. AJR Am J Roentgenol 2012;199:W17-23.

11. Lee YL, Huang YK, Hsu LS, Chen PY, Chen CW. The use of non-contrast-enhanced MRI to evaluate serial changes in endoleaks after aortic stenting: a case report. BMC Med Imaging 2019;19:82.

12. Hsu YC, Huang YK, Hsu LS, Chen PY, Chen CW. Using non-contrast-enhanced magnetic resonance venography

Cite this article as: Chen CW, Lin KH, Yang TY, Chen PY, Hsu YC, Huang YK. Usefulness of non-contrast-enhanced magnetic resonance venography in assessing Klippel-Trenaunay syndrome in an adult patient with renal insufficiency: a case description. Quant Imaging Med Surg 2022;12(3):2193-2198. doi: 10.21037/qims-21-671 for the evaluation of May-Thurner syndrome in patients with renal insufficiency: A case report. Medicine (Baltimore) 2019;98:e18427.

13. Lim RP, Hecht EM, Xu J, Babb JS, Oesingmann N, Wong S, Muhs BE, Gagne P, Lee VS. 3D nongadoliniumenhanced ECG-gated MRA of the distal lower extremities: preliminary clinical experience. J Magn Reson Imaging 2008;28:181-9. 


\section{Supplementary}

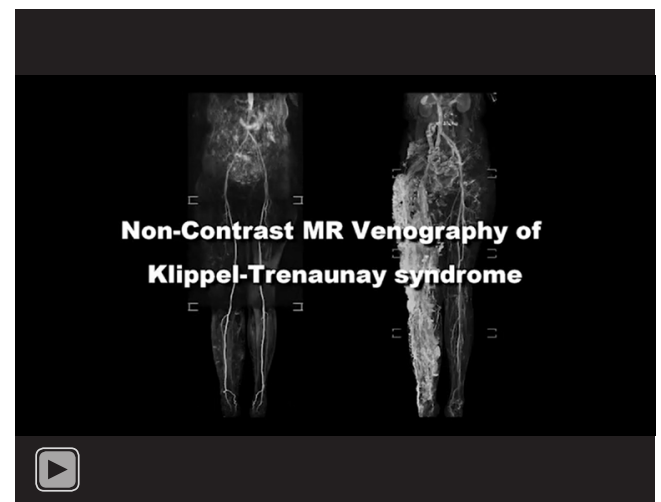

Video S1 The video that demonstrates the three-dimensional rotating projection of the affected extremity showing femoral vein aplasia and extensive venous malformations. 